\title{
Classification and Acquisition of Contradictory Event Pairs using Crowdsourcing
}

\author{
Yu Takabatake ${ }^{1}$ \\ Hajime Morita ${ }^{1,2} \quad$ Daisuke Kawahara $^{1}$ \\ Sadao Kurohashi $^{1,2} \quad$ Ryuichiro Higashinaka $^{3} \quad$ Yoshihiro Matsuo $^{3}$ \\ ${ }^{1}$ Kyoto University ${ }^{2}$ CREST, Japan Science and Technology Agency \\ ${ }^{3}$ NTT Media Intelligence Laboratories \\ takabatake@nlp.ist.i.kyoto-u.ac.jp, \{hmorita,dk, kuro\}@i.kyoto-u.ac.jp, \\ \{higashinaka.ryuichiro, matsuo.yoshihiro\}@lab.ntt.co.jp
}

\begin{abstract}
We propose a taxonomy of contradictory event pairs and a method for building a database of such pairs. When a dialog system participates in an open-domain conversation with a human, it is important to avoid the generation of utterances that conflict with the context of the dialog. Here, we refer to a pair of events that are not able to co-occur or that are not inconsistent with each other as a contradictory event pair. In this study, we collected contradictory event pairs using crowdsourcing and constructed a taxonomy of such pairs. We also built a large-scale database of Japanese contradictory event pairs for each class using crowdsourcing. This database will be used for consistent utterance generation in dialog systems.
\end{abstract}

\section{Introduction}

In recent years, speech dialog systems such as Siri have become widespread. A user of such systems can obtain weather and traffic information by speaking to his/her smartphone. Although such information-seeking applications are now used in our daily lives, natural open-domain conversations are also expected.

Current open-domain conversation functions not only repeat single isolated utterances, but can also maintain previous dialog logs and conduct multitern conversations. However, many technical problems must be solved to generate natural utterances in the context of conversations.

In open-domain conversations, it is necessary to talk about related things. Such relatedness can be automatically learned from a large corpus, but a simple strategy of saying related things can lead to a nonsensical utterance as follows:

U: I like traveling, but I've never been to Paris.

S: Have you ever climbed the Eiffel Tower?

In this conversation, the system utterance $(\mathrm{S})$ was generated using the keyword "Eiffel Tower," which is related to "Paris" in the user utterance (U). However, because "having never been to Paris" and "having climbed the Eiffel Tower" are contradictory, this system utterance is totally unacceptable. To conduct natural conversations, it is necessary to cope with contradictory phenomena.

In the field of natural language processing, contradictions have been dealt with in recognizing textual entailment (RTE), but there have been no studies that classify contradictory phenomena, to the best of our knowledge. In this study, we collected contradictory event pairs using crowdsourcing and constructed a taxonomy of such pairs. Furthermore, based on the contradiction taxonomy, we built a large-scale database of Japanese contradictory event pairs using crowdsourcing.

\section{Related Work}

There are several studies related to this research, including recognition and acquisition of contradictory event pairs (Harabagiu et al., 2006; Hashimoto et al., 2012; Kloetzer et al., 2013), acquisition of world knowledge (Fujita et al., 2014; Inui et al., 2005), and quality control of crowdsourced products (Whitehill et al., 2009). In contrast, we construct a taxonomy of contradictory event pairs, and thus the aim is different. However, our taxonomy could assist 
the recognition and acquisition methods proposed in these studies.

\subsection{Recognition of Contradictory Event Pairs}

As mentioned above, the recognition of contradictory event pairs is related to RTE. In some RTE tasks, contradiction is one of the relations between text and hypothesis. For example, Harabagiu et al. (2006) proposed a method to recognize contradictions between texts using negation expressions, antonyms, and discourse analysis.

Recognition of contradictory event pairs plays an important role in the systems that detect contradictions between information extracted from web texts. For example, there are several systems that detect contradictory information, such as WISDOM (Akamine et al., 2009), Statement Map (Murakami et al., 2009), and Dispute Finder (Ennals et al., 2010).

\subsection{Acquisition of Contradictory Event Pairs}

Hashimoto et al. (2012) and Kloetzer et al. (2013) proposed methods for acquiring contradictory event pairs. Hashimoto et al. (2012) collected Japanese contradictory and consistent event pairs using templates of semantic polarities that indicate excitatory, inhibitory, and neutral properties. A template consists of a particle and a predicate, such as “を (particle) 破壊する (destroy)” and “を (particle) 進行させ る (develop)." They collected one million contradictory event pairs, such as “ガンを破壊する (destroy cancer)” and “ガンを進行させる (develop cancer),” with a precision of $70 \%$ in their experiments. Most of the pairs were categorized as simultaneous contradiction, in particular, as continuous or binary contradiction in our taxonomy (described later). Kloetzer et al. (2013) refined this method and collected 75,000 contradictory event pairs with a precision of $80 \%$.

\subsection{Construction of World Knowledge}

Fujita et al. (2014) and Inui et al. (2005) proposed a method for constructing world knowledge. Both methods focus on causal knowledge among world knowledge and automatically collected such knowledge for Japanese. Fujita et al. (2014) extracted text fragments preceding and following the conjunction “のに (but)," aiming to collect expressions indicating events that are unexpected by the author of the text. They finally extracted a small number of causal event pairs (approximately 1,400 pairs) from community question answering texts.

Inui et al. (2005) defined four types of causal relations between events based on whether the event is an action or a situation. They classified sentences that included the conjunction “ため (because)" into the four types of relations. They achieved a precision of over $95 \%$ with a recall of $80 \%$ for three types of relations ("cause," "precondition" and "means"), and achieved a precision of $90 \%$ and a recall of $30 \%$ for the fourth relation, "effect."

\subsection{Quality Control of Crowdsourced Products}

There are two common approaches to quality control of crowdsourced products. Whitehill et al. (2009) proposed a probabilistic method for combining the labels of multiple crowdworkers to acquire reliable labels. Their method outperforms the commonlyused majority voting. The second approach is to measure the reliability of crowdworkers using gold standard data. We used both approaches to build a large-scale database of contradictory event pairs via crowdsourcing.

\section{Taxonomy of Contradictory Event Pairs}

\subsection{Collecting Contradictory Event Pairs}

To construct a taxonomy of contradictory event pairs, we need to investigate real examples of such pairs. To obtain a large number and variety of contradictory event pairs, we employed crowdsourcing, which can employ a large number of people to perform micro-tasks inexpensively and over a short period of time. We used Yahoo! crowdsourcing ${ }^{1}$ as a crowdsourcing service.

Considering future applications of the resulting taxonomy in open-domain dialog systems, we focused on two domains: "gourmet" and "traveling." We first prepared sentences referring to events specific to each domain. Presenting the domain and one of its specific sentences (hereafter, called a target sentence), we asked crowdworkers to write contradictory sentences. We asked crowdworkers to avoid writing self-contradictory sentences such as "The sun rises in the west." as well as sentences that are unrelated to the target sentence, such as "It's my

\footnotetext{
${ }^{1}$ http://crowdsourcing.yahoo.co.jp/
} 
birthday today." for the target sentence "It's raining today."

We prepared 15 target sentences for each domain, and asked 20 crowdworkers to write five contradictory sentences for each target sentence. That is, we collected 100 contradictory sentences for each target sentence. For example, presenting the domain "gourmet" and the target sentence "I love Chinese cuisine," we obtained contradictory sentences such as "I don't like Chinese cuisine." and "I've eaten only Japanese cuisine."

\subsection{Basic Idea}

When classifying collected contradictory event pairs, the interpretation of these sentences can be a problem. For example, the event pair /being in Tokyo, being in Kyoto $\rangle$ is not contradictory if the events happen at different times, but is contradictory when they happen simultaneously. The event pair $\langle$ the pasta tastes delicious, he didn't eat the pasta $\rangle$ is not contradictory if the subject knew that the pasta was delicious but did not eat it for some other reason. However, for the event pair $\langle$ he ate the pasta, and the pasta tasted delicious $\rangle$, if the former event is "he didn't eat the pasta," such an event pair is contradictory because the subject cannot know that the pasta that he did not eat tasted delicious.

Therefore, we do not interpret the collected event pair sentences, but instead examine the possible variations of contradictory phenomena between these event pairs by only referring to the original sentences.

As discussed above, it is necessary to discriminate whether an event pair occurs simultaneously or has a temporal or order relation to judge if it is contradictory. We call the former type a simultaneous contradiction, such as $\langle$ being in Tokyo, being in Kyoto $\rangle$. We call the latter type a transitional contradiction, such as $\langle$ he didn't eat the pasta, the pasta tasted delicious $\rangle$. In this way, we first classify contradictory event pairs into simultaneous contradictions and transitional contradictions.

The rest of this section further classifies both simultaneous contradictions and transitional contradictions. Table 1 summarizes our taxonomy of contradictory event pairs and lists examples.

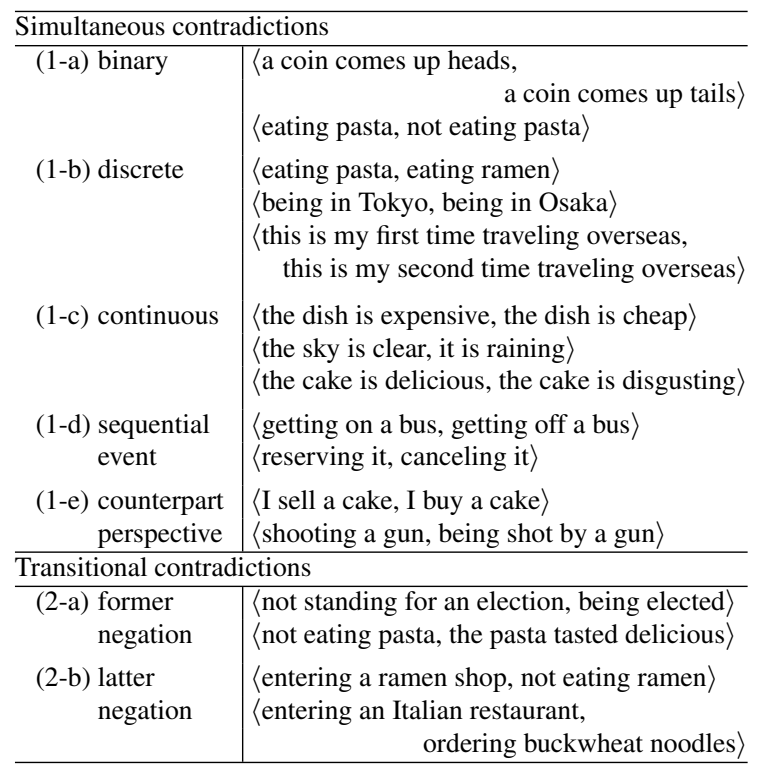

Table 1: Our taxonomy of contradictory event pairs with examples. Examples are translated into English.

\subsection{Simultaneous Contradictions}

When a pair of two events occurs simultaneously, contradictions of such pairs have a strong relation with negation, such as having a meal, not having a meal $\rangle$ and 〈eating to excess, eating moderately . There are also contradictory event pairs based on sibling relations, such as $\langle$ being in Tokyo, being in Kyoto〉, where "Tokyo" and "Kyoto" have a sibling relation. We therefore classify negation and sibling relations into binary (e.g., "single" and "married"), discrete (e.g., "Tokyo" and "Kyoto"), and continuous (e.g., "expensive" and "cheap").

Furthermore, negation has the following two classes that can cause contradictions (Izumi et al., 2014): sequential event relations, such as "getting on" and "getting off," and counterpart perspective relations, such as "selling" and "buying." We added these classes to our taxonomy. The subclasses of simultaneous contradictions are detailed below.

1-a. binary When an event pair includes mutually exclusive antonyms (e.g., "single" and "married") or a predicate and its negation (e.g., "going" and "not going"), these events are contradictory. We call such contradictory event pairs binary.

1-b. discrete When an event pair consists of predicates or arguments that have sibling relations, such as $\langle$ being in Tokyo, being in Kyoto $\rangle$ and 
〈eating pasta, eating ramen $\rangle$, these events are contradictory. $^{2}$ We call such contradictory event pairs discrete pairs to discriminate them from the next class, "continuous." We also include contradictory event pairs caused by numerical values in the discrete class such as $\langle$ this is my first time traveling overseas, this is my second time traveling overseas $\rangle$.

1-c. continuous When an event pair consists of antonym predicates that represent continuous states, such as "expensive" and "cheap," these events are contradictory. We call such contradictory event pairs continuous. In addition to $\langle$ being expensive, being cheap $\rangle$, $\langle$ being expensive, not being expensive $\rangle$ and $\langle$ being extremely expensive, being a little expensive $\rangle$ are also contradictory event pairs. Since continuous states are not necessarily one-dimensional, various continuous event pairs can be contradictory, such as 〈it's clear, it's raining , $\langle$ it's clear, it's stormy $\rangle$, and 〈it's snowing, it's raining $\rangle$.

1-d. sequential event relations Sequential event pairs, such as (getting on a bus, (and then) getting off a bus $\rangle$, are not contradictory if time goes by between the two events. However, when we consider /getting on a bus, getting off a bus $\rangle$ as a pair of simultaneous events, this event pair is contradictory.

\section{1-e. counterpart perspective relations}

Counterpart perspective events such as $\langle\mathrm{I}$ sell a book to him, he buys a book from me $\rangle$ are not contradictory if these event descriptions indicate the same event from a different view. However, when we consider $\langle$ I sell a book to him, I buy a book from him $\rangle$ as a simultaneous event with the same participants, this event pair is contradictory.

For an event pair of simultaneous contradictions, event pairs where one of the events is replaced by its synonymous event are also regarded as simultaneous contradictions. For example, 〈tasting delicious, not tasting delicious $\rangle$ is a "simultaneous contradic-

\footnotetext{
${ }^{2}$ Event pairs that have a sibling relation in their arguments are not always contradictory, as in $\langle$ I like Toyotas, I like Nissans $\rangle$.
}

tion: continuous," and thus $\langle$ tasting nice, not tasting delicious $\rangle$, where "tasting delicious" is synonymous with "tasting nice," is also classified as a "simultaneous contradiction: continuous."

\subsection{Transitional Contradictions}

Transitional contradictions are a relation between two events that have a temporal or order relation. We call the event that happens earlier the former event and the event that happens later the latter event, and represent this relation as $\mathrm{A} \sim \mathrm{B}$. When $\mathrm{A}$ and $\mathrm{B}$ have such a temporal or order relation, a pair of $\langle\neg A, B\rangle$ or $\langle A, \neg B\rangle$ is contradictory if one of the following conditions is satisfied.

2-a. former negation Under $A \sim B$, there is a case such that $\mathrm{A}$ is a precondition for B. For example, for the event pair 〈having a passport, going overseas $\rangle$, the precondition for "going overseas" is "having a passport." For such a relation, the negation of the precondition $(\neg \mathrm{A})$ is contradictory to B. For the passport example, 〈not having a passport, going overseas〉 is contradictory.

2-b. latter negation Under $A \sim B$, when $A$ generally leads $\mathrm{B}$, the negation of $\mathrm{B}$ is contradictory to A. For example, the event "entering a restaurant" generally leads to the event "ordering something." Accordingly, <entering a restaurant, not ordering something $\rangle$, which is made by negating $\mathrm{B}$, is contradictory.

The above classification for $A \leadsto B$ is based on a temporal or order relation between $\mathrm{A}$ and $\mathrm{B}$. This can be also interpreted from another viewpoint as follows. For the former negation class of $A \sim B$, it generally holds that if $\mathrm{B}$ is true, $\mathrm{A}$ is true as a precondition (e.g., "if going overseas, a traveler has a passport"), and A is negated to be a contradictory event pair. For the latter negation class of $A \sim B$, it generally holds that if A is true, B is true, and negating B leads to be a contradictory event pair. That is to say, the above classes are the negation of the former event and the latter event, respectively, from the viewpoint of a temporal or order relation, but they are also the negation of consequences from the viewpoint of an if-then relation.

For transitional contradictions, it is difficult to define the extent of generally accepted common sense. 
If we suppose real-world event pairs, it is very rare that if-then relations absolutely hold. That is, we can easily think of a counterexample that does not satisfy an if-then relation. Furthermore, if there are some cases that do not meet an if-then relation, we do not believe that its former negation or latter negation is contradictory. For example, we can easily think of counterexamples for "if going to a spa, he/she wears a summer kimono," and thus we do not believe that the event pair going to a spa, not wearing a summer kimono $\rangle$ is contradictory.

\subsection{Multistage Inference Contradictions}

There are contradictory event pairs based on multistage inferences. For example, $\langle$ I made a supper using leftovers, I had a full course dinner $\rangle$ is a contradictory event pair. First, we can generally accept the following event relations: 〈I made a supper using leftovers, I ate a few dishes for supper $\rangle$ and $\langle\mathrm{I}$ had a full course dinner, I ate many dishes for dinner. Here, $\langle\mathrm{I}$ ate a few dishes for supper, I ate many dishes for dinner $\rangle$ is classified as a "simultaneous contradiction: continuous" (1-c), and thus $\langle$ I made a supper using leftovers, I had a full course dinner $\rangle$ is a result of these inferences.

\section{Data Construction by Crowdsourcing}

We constructed domain-specific contradictory event pairs using crowdsourcing, exploiting their classifications explained in the previous section. Considering the application to open-domain conversational systems, we selected 12 domains: "gourmet," "travel," "weather," "sports," "life," "political and economic," "child-rearing," "learn," "health," "work," "baseball," and "shopping."

To construct the data, we presented a domain to crowdworkers and asked them to create domainspecific contradictory event pairs. However, it is actually difficult to make this a single complete task. In crowdsourcing, a task is supposed to be relatively simple so that it may be done in a short time by ordinary people. The task explanation is also ought to be simple and quickly understandable. A complex task that requires a long explanation increases the load on crowdworkers and makes it difficult for the task to be accurately completed.

In this study, the process of constructing contradictory event pairs was divided into two phases, the construction of a domain-specific sentence and construction of its contradictory sentence. Furthermore, each phase was completed using a task comprising two stages: construction and evaluation. That is, the process of constructing contradictory event pairs consisted of the following four crowdsourcing tasks:

- Phase 1: Construction of domain-specific sentences (target sentences).

- Phase 2: Evaluation of target sentences to determine if they are actually suitable for each domain.

- Phase 3: Construction of contradictory sentences for each target sentence.

- Phase 4: Evaluation of each pair of domainspecific and contradictory sentences to determine if they are really contradictory.

Here, by using the classifications of contradictory event pairs in Phase 3 and 4, we make the task clearer and more simple (Sections 4.3 and 4.4 explain the details).

\subsection{Phase 1: Construction of Domain-specific Sentences (Target Sentences)}

Crowdworkers were shown a domain and were asked to submit sentences that express domainspecific events. For the music domain, the following examples of domain-specific events were shown to crowdworkers: "a guitar is too noisy" and "I bought high-quality earphones." To make Phase 3 easier, types of inappropriate sentences and instructions for revising them were also presented to crowdworkers.

- Nominals: "performance of instruments" $\rightarrow$ "I play instruments"

- Pronouns: "It's fun to play it" $\rightarrow$ "It's fun to play a violin"

- Monologue: "It's jazz!" $\rightarrow$ "I listen to jazz"

- Call/Invitation: "Let's go to a concert" $\rightarrow$ "I would like to go to a concert"

For each domain (out of 12 domains), we asked 100 crowdworkers to submit five sentences, that is, we constructed 6,000 target sentences in total.

\subsection{Phase 2: Evaluation of Target Sentences}

We asked crowdworkers whether the target sentence constructed in Phase 1 was really domain-specific and met all the following criteria. Counter-examples (CE) for each criterion in the domain of music are shown below. 
- It makes sense.

CE: "a component of a band is necessity to sell a music."

- It is domain-specific.

CE: "These noodles are delicious."

- It is a sentence (not just a noun or verb)

CE: "chorus", "enjoy."

- It is not a noun phrase

CE: "favorite guitar", "performance of instruments."

- Not including a pronoun

CE: "it's fun to play it."

- It is not a remark

CE: "it's a jazz!"

- It is a call/invitation

CE: "let's go to a concert"

- It is a interrogative

CE: "Do you like a classic music?"

We asked five crowdworkers to evaluate for each of the target sentences in 6,000 sentences obtained in Phase 1. For each domain, approximately 200 sentences received all positive evaluations. Based on this result, for each domain, we selected the top 200 target sentences to use in Phase 3. To select these top sentences, we used Whitehill's EM-based evaluation method (Whitehill et al., 2009), which is widely used for the evaluation of crowdsourcing results.

\subsection{Phase 3: Construction of Contradictory Sentences}

Our preliminary experiments found that it is difficult to collect transitional contradictions by just asking crowdworkers to write "some contradictory sentences." To solve this problem, we divided the task into three smaller tasks to create, simultaneous contradictions, former-negative transitional contradictions, and latter-negative transitional contradictions.

\section{Simultaneous contradictions}

We asked crowdworkers to write simultaneous contradictory sentences for a given target sentence. To help workers understand the concept of simultaneous contradiction, the following examples, based on the taxonomy described in Section 3, were shown.

- Opposite meaning: 〈a coin comes up heads, a coin comes up tails $\rangle$ or, 〈eating pasta, not eating pasta〉
- Same category, but different: 〈eating pasta, eating ramen $\rangle$ or, 〈I'm in Tokyo, I'm in Osaka $\rangle$

- Difference in degree: 〈the cake is delicious, the cake is disgusting or, 〈it's clear, it is raining

- Different role: 〈I sell a cake, I buy a cake〉 or, 〈he shot a gun, he was shot by a gun〉

- Simultaneous occurrence of naturally sequential events: 〈getting on a bus, getting off a bus〉 or, 〈enrolling in a school, graduating from a school $\rangle$

The following example contradictory phrases for "saving money" were shown to crowdworders: "saving stamps" and "spending money." The following cautions were also given:

- Do not write a sentence that is impossible by itself, such as "The sun rises in the West"

- Do not write a sentence that is totally unrelated to a given target sentence, such as "It's my birthday today." for "It's raining."

For each domain (out of 12 domains), we prepared 200 target sentences. For each target sentence, we asked 10 crowdworkers to submit more than one contradictory sentence. As a result, after discarding inappropriate sentences (such as single word sentences) and merging identical sentences, we obtained 14 contradictory sentences for each target sentence on average.

\section{Former-negative transitional contradictions}

A former-negative transitional contradiction is the relation between an event and the negation of its precondition. For crowdworkers, however, it is easier to consider preconditions for a target sentence than, consider the precondition negations. Therefore, we asked crowdworkers to write preconditions to a given target sentence.

As example of preconditions for "being elected," "standing for election" and "being eligible for election" were shown to crowdworkers. The following cautions were also given to them.

- Do not write an unrelated or mostly un-related sentence for a given target sentence, such as "there is air." to "The candidate is elected."

For each domain (out of 12 domains), we prepared 200 target sentences. For each target sentence, we asked 10 crowdworkers to submit more than one contradictory sentence. As a result, we obtained 17 precondition sentences for each target sentence on average. 


\section{Latter-negative transitional contradictions}

A latter-negative transitional contradiction is the relation between an event and the negation of the event that generally follows it. However, similarly to the case of former-negative transitional contradictions, it is easier for crowdworkers to consider generally-following events than their negations. Therefore, we asked crowdworkers to write sentences that generally follow a given target sentence. We modified target sentences to be past tense in order to emphasize that former events expressed by the target sentences had already happened, and that we were interested in the following events.

As example events that generally follow "I went to a hot spring.", "I wash one's body." and "I soak in a hot spring." were shown to crowdworkers. The following cautions were also given to them.

- Do not write a sentences that is unrelated or mostly unrelated to a given target sentence, such as "The next day comes" for "He stood as a candidate."

Similarly to the case of former-negative transitional contradictions, we obtained 17 generallyfollowing sentences for each target sentence on average.

\subsection{Phase 4: Evaluation of Contradictory Sentence Pairs}

Evaluations using crowdsourcing were performed for each of the three categories.

\section{Simultaneous contradictions}

We asked crowdworkers if a pair of the target and contradictory sentences was really contradictory.

We asked five crowdworkers to evaluate for each of the 34,900 simultaneous contradiction pairs obtained in Phase 3. As a result, $77 \%$ of the pairs received more than two positive answers.

\section{Former-negative transitional contradictions}

We modified the preconditions collected in Phase 3 into a negative form automatically. We then asked crowdworkers if a pair of target sentence and its negated precondition was really contradictory.

We asked five crowdworkers to evaluate each pair in 41,300 former-negative transitional contradiction pairs. As a result, $49 \%$ of the pairs received more than two positive answers.

\begin{tabular}{|c|c|}
\hline \multicolumn{2}{|c|}{ simultaneous contradiction } \\
\hline binary & $\begin{array}{l}\langle\text { going to Tokyo by plane, } \\
\quad \text { going to Tokyo, but not by plane }\rangle \\
\langle\text { making a plan, planning nothing }\rangle\end{array}$ \\
\hline discrete & $\begin{array}{r}\langle\text { going to Las Vegas on vacation, } \\
\text { going to Hawaii on vacation }\rangle \\
\langle\text { going to Tokyo by air, going to Tokyo by train }\rangle\end{array}$ \\
\hline continuous & $\begin{array}{l}\langle\text { I find overseas travel hard, } \\
\text { I find overseas travel easy }\rangle \\
\langle\text { the guide was very kind, the guide was unkind }\rangle\end{array}$ \\
\hline $\begin{array}{l}\text { sequential event } \\
\text { relations }\end{array}$ & $\begin{array}{l}\langle\text { checking into a hotel, checking out of a hotel }\rangle \\
\langle\text { booking a tour abroad, }\end{array}$ \\
\hline $\begin{array}{l}\text { counterpart perspective } \\
\text { relations }\end{array}$ & $\langle$ staying at a hotel, accomodating a guest $\rangle$ \\
\hline \multicolumn{2}{|c|}{$\begin{array}{l}\text { Transitional Contradictions } \\
\end{array}$} \\
\hline former negation & $\begin{array}{c}\langle\text { not buying flight ticket, } \\
\text { going to Tokyo by plane }\rangle \\
\langle\text { not applying for a passport, } \\
\text { going to Las Vegas on vacation }\rangle \\
\langle\text { not arriving at a hotel, checking into a hotel }\rangle \\
\langle\text { I have never traveled abroad, } \\
\text { I find hard overseas travel difficult }\rangle\end{array}$ \\
\hline latter negation & $\begin{array}{c}\langle\text { booking a tour abroad, } \\
\text { not applying for a passport }\rangle \\
\text { (going to Tokyo by plane, } \\
\text { not landing at the airport }\rangle \\
\text { (going to Las Vegas to play, } \\
\quad \text { not taking a plane trip }\rangle \\
\langle\text { staying at a hotel, not receiving a room key }\rangle\end{array}$ \\
\hline
\end{tabular}

Table 2: Examples of contradictory event pairs obtained by crowdsourcing. Examples have been translated into English.

\section{Latter-negative transitional contradictions}

We modified the generally-following sentences collected in Phase 3 into a negative form automatically. We then asked crowdworkers if a pair of the target sentence and its negated generally-following sentence is really contradictory.

We asked five crowdworkers to evaluate for each of the 42,080 latter-negative transitional contradiction pairs. As a result, 37\% of the pairs received more than two positive answers.

\subsection{Discussion}

As a result of the series of crowdsourcing tasks, we constructed 118,380 contradictory event pairs, each of which has been evaluations by five crowdworkers.

To make the crowdsourcing tasks clearer, we divided the tasks into three categories according to our taxonomy. However, we sometimes obtained missclassified contradictory pairs in each category. For example, we acquired $\langle$ I have no credit card, I buy something with my credit card $\rangle$ as a simultaneous contradiction, but we classify it as a former-negative 


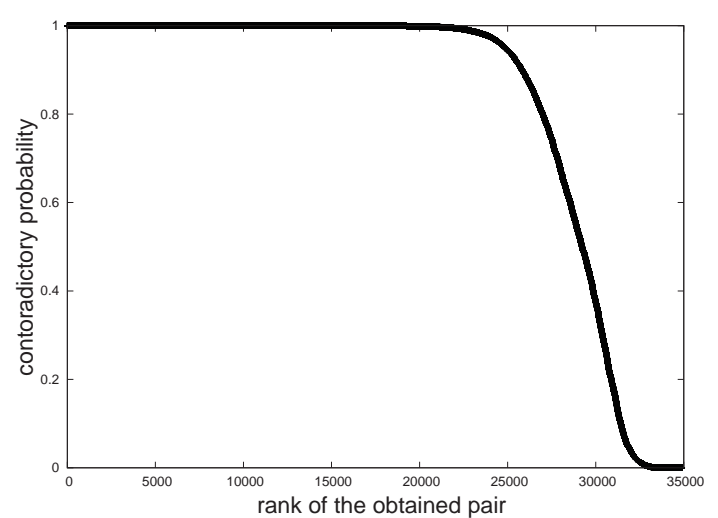

Figure 1: Contradictory probabilities distribution for simultaneous contradiction pairs.

transitional contradiction.

The distributions of contradictory probabilities of the constructed pairs calculated by the Whitehill's EM-based method is shown in Figures 1, 2 and 3. Because negation, antonym and sibling relation are relatively clear, we were able to obtain high-quality data for simultaneous contradiction. However, the number of contradictory event pairs with a high probability became smaller for former-negative transitional contradictions, and much smaller for latternegative ones.

In the case of latter-negative transitional contradictions, for example, 〈the Japanese legislature was dissolved, there are no breaking news stories $\rangle$ has a probability of 0.8 , and it can be considered as a widely-acceptable contradictory event pair. However, $\langle$ I watched a baseball broadcast, I did not enjoy the baseball game $\rangle$ has a probability of 0.5 and we feel that it is not necessarily a contradiction. Because the judgment of a transitional contradiction is based on common knowledge or the life-style of each person, its contradictory probabilitiy often decreases.

\section{Conclusion}

In this paper, we proposed a taxonomy of contradictory event pairs. We first discriminated between simultaneous contradictions and transitional contradictions, and then classified these further. The event pair $\langle$ having never been to Paris, having climbed the Eiffel Tower), which was mentioned in Section 1, is classified as a "transitional contradiction: former negation" (2-a).

Based on our taxonomy, we built a large-scale database of Japanese contradictory event pairs for

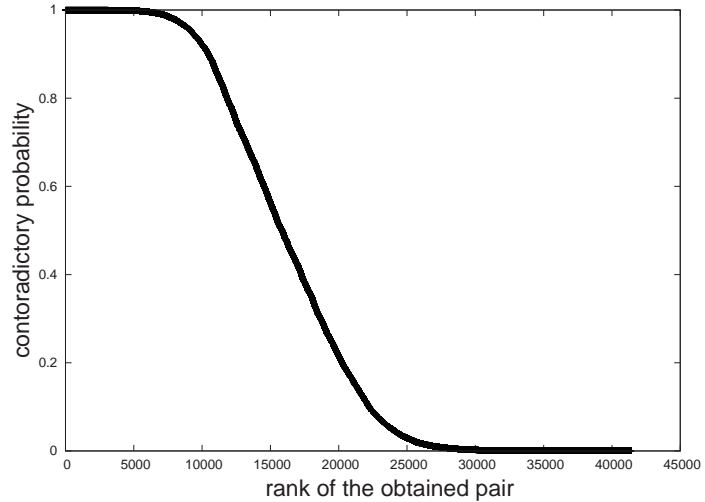

Figure 2: Contradictory probabilities distribution for former-negative transitional contradiction pairs.

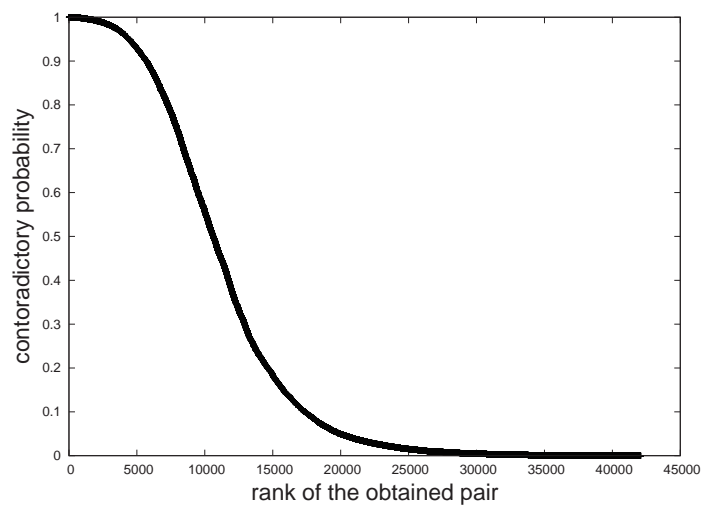

Figure 3: Contradictory probobility distribution for latternegative transitional contradiction.

each class using crowdsourcing. As a result, we obtained more than 100,000 possible contradictory event pairs in total, and out of these, over 60,000 event pairs can be accepted as contradictory event pairs based on the evaluations of crowdworkers.

In the future, we intend to develop an opendomain conversational system that does not generate contradictory utterances on the basis of the acquired contradiction database. 


\section{References}

Susumu Akamine, Daisuke Kawahara, Yoshikiyo Kato, Tetsuji Nakagawa, Kentaro Inui, Sadao Kurohashi, and Yutaka Kidawara. 2009. WISDOM: A web information credibility analysis system. In Proceedings of ACL-IJCNLP2009 Software Demonstrations, pages 14.

Rob Ennals, Beth Trushkowsky, and John Mark Agosta. 2010. Highlighting disputed claims on the web. In Proceedings of the 19th international conference on World wide web, pages 341-350. ACM.

Hiroshi Fujita, Akira Fujita, and Naoyoshi Tamura. 2014. Extracting implicit causal knowledge using the conjunctive marker noni (in Japanese). IEICE technical report. Natural language understanding and models of communication, 114(366):61-66, dec.

Sanda Harabagiu, Andrew Hickl, and Finley Lacatusu. 2006. Negation, contrast and contradiction in text processing. In Proceedings of $A A A I$, volume 6, pages 755-762.

Chikara Hashimoto, Kentaro Torisawa, Stijn De Saeger, Jong-Hoon Oh, and Jun'ichi Kazama. 2012. Excitatory or inhibitory: A new semantic orientation extracts contradiction and causality from the web. In Proceedings of EMNLP2012, pages 619-630.

Takashi Inui, Kentaro Inui, and Yuji Matsumoto. 2005. Acquiring causal knowledge from text using the connective marker tame. ACM Transactions on Asian Language Information Processing (TALIP), 4(4):435474.

Tomoko Izumi, Tomohide Shibata, Hisako Asano, Yoshihiro Matsuo, and Sadao Kurohashi. 2014. Constructing a corpus of Japanese predicate phrases for synonym/antonym relations. In Nicoletta Calzolari, Khalid Choukri, Thierry Declerck, Hrafn Loftsson, Bente Maegaard, Joseph Mariani, Asuncion Moreno, Jan Odijk, and Stelios Piperidis, editors, Proceedings of the Ninth International Conference on Language Resources and Evaluation (LREC'14), pages 1394-1400, Reykjavik, Iceland, May. European Language Resources Association (ELRA). ACL Anthology Identifier: L14-1244.

Julien Kloetzer, Stijn De Saeger, Kentaro Torisawa, Chikara Hashimoto, Jong-Hoon Oh, Motoki Sano, and Kiyonori Ohtake. 2013. Two-stage method for largescale acquisition of contradiction pattern pairs using entailment. In Proceedings of EMNLP2013, pages 693-703.

Koji Murakami, Eric Nichols, Suguru Matsuyoshi, Asuka Sumida, Shouko Masuda, Kentaro Inui, and Yuji Matumoto. 2009. Statement map: Assisting information crediblity analysis by visualizing arguments. In Proceedings of the 3rd Workshop on Information Credibility on the web, pages 43-50. ACM.
Jacob Whitehill, Ting fan Wu, Jacob Bergsma, Javier R. Movellan, and Paul L. Ruvolo. 2009. Whose vote should count more: Optimal integration of labels from labelers of unknown expertise. In Y. Bengio, D. Schuurmans, J.D. Lafferty, C.K.I. Williams, and A. Culotta, editors, Advances in Neural Information Processing Systems 22, pages 2035-2043. Curran Associates, Inc. 